\title{
Development of clinical care guidelines for faculty and residents in the era of COVID-19
}

\author{
Steven D. Pletcher, MD ${ }^{1}$ \\ ${ }^{1}$ University of California San Francisco School of Medicine
}

April 28, 2020

\begin{abstract}
Background The global COVID-19 pandemic brings new challenges to otolaryngology resident education. Surgical volume and clinic visits are curtailed, personal protective equipment for operating room participation is restricted, and the risk of COVID-19 disease transmission during heretofore routine patient care is the new norm. Methods We describe a small-team "cohorting" protocol including guidelines for faculty and resident in common clinical scenarios with attention paid to the risk of common otolaryngologic procedures. Results A rotating small-team approach was implemented at each clinical site, limiting interaction between Department members but providing comprehensive coverage. Faculty were involved at the earliest phase of clinical interactions. Guidelines delineated faculty and resident roles based on risk stratification by patient COVID status and anticipated procedures. Special consideration was given to high-risk procedures such as endoscopy and tracheotomy. Conclusions A small-team based approach with guidelines for faculty/resident roles may mitigate risk while optimizing patient care and maximizing education.
\end{abstract}

Abstract content goes here

Jason S. Park, MD, $\mathrm{PhD}^{1}$; Ivan H. El-Sayed, $\mathrm{MD}^{1}$; VyVy N. Young, $\mathrm{MD}^{1}$; Steven D. Pletcher, $\mathrm{MD}^{1, *}$

${ }^{1}$ Department of Otolaryngology-Head and Neck Surgery, School of Medicine, University of California San Francisco, San Francisco, CA, USA

${ }^{*}$ Corresponding author: Steven D. Pletcher, MD Professor, Residency Program Director Department of Otolaryngology - Head and Neck Surgery University of California San Francisco School of Medicine 2233 Post Street, $3^{\text {rd }}$ Floor, San Francisco, CA 94115 e-mail: Steven.Pletcher@ucsf.edu

No grant support to report. This work has not been presented at any meetings or conferences.

Brief running title:

COVID-19 era guidelines for faculty and residents

Key words:

COVID-19, guidelines, cohorting, residency, trainee, education, aerosol-generating procedures

Abstract

Background

The global COVID-19 pandemic brings new challenges to otolaryngology resident education. Surgical volume and clinic visits are curtailed, personal protective equipment for operating room participation is restricted, and the risk of COVID-19 disease transmission during heretofore routine patient care is the new norm.

Methods 
We describe a small-team "cohorting" protocol including guidelines for faculty and resident in common clinical scenarios with attention paid to the risk of common otolaryngologic procedures.

\section{Results}

A rotating small-team approach was implemented at each clinical site, limiting interaction between Department members but providing comprehensive coverage. Faculty were involved at the earliest phase of clinical interactions. Guidelines delineated faculty and resident roles based on risk stratification by patient COVID status and anticipated procedures. Special consideration was given to high-risk procedures such as endoscopy and tracheotomy.

\section{Conclusions}

A small-team based approach with guidelines for faculty/resident roles may mitigate risk while optimizing patient care and maximizing education.

\section{Introduction}

Even in the best of times, providing excellent resident education involves many challenges. Ensuring outstanding surgical care while training residents in surgery, maintaining appropriate balance between service and education, and designing a graduated experience that allows a natural transition of trainees from Resident to Faculty are some of the baseline conundrums faced by all surgical training programs. The introduction of the global COVID-19 pandemic has disrupted our practices and added additional dimensions to these challenges.

As Educators, our first step in responding to this pandemic must be to understand its potential areas of impact on our trainees. Hands-on surgical exposure for trainees has been dramatically curtailed by a decreased volume of surgical procedures. On March $23^{\text {rd }}$, the American Academy of Otolaryngology Head and Neck Surgery officially recommended limiting patient interactions to "time-sensitive and emergent problems" and resident surveys demonstrate that, during the same time frame, nearly all programs surveyed had enacted policy changes to decrease COVID-19 spread $^{1,2}$. Additionally, risk of infection restrictions on personal protective equipment (PPE) can limit operating room participation to only essential personnel.

The role of the head and neck surgeon and trainees with whom we work, however, extends beyond the operating room. Much of the risk we face is presented during inpatient management and consultations. Training and patient care in this environment presents a different set of challenges. The "routine" examinations and procedures that make up much of the daily life of a resident now require a careful assessment of risk to trainees, faculty, and staff. Medicine is a team sport and we also face the risk of infecting each other. Early results from contact tracing of infected health care workers at our institution demonstrate that transmission between health care providers is almost as likely as transmission from patients ${ }^{3}$. Many clinics have started using telehealth for initial ambulatory visits and limiting the people in the room during clinic assessments to mitigate ambulatory risk, but in-hospital consultations remain a source of concern.

The COVID-19 pandemic required restructuring of faculty and resident assignments to protect the safety of healthcare workers and patients alike. In this paper, we describe the creation and implementation of guidelines delineating resident and faculty responsibilities in accordance with safety mandates to reduce the risk (to providers, other health care workers, and patients) while preserving resident education.

\section{Methods}

We describe the conception and implementation of a small-team "cohorting" protocol. A literature search was performed to assess and categorize risk of common procedures performed by Otolaryngology - Head and Neck Surgery residents. An iterative process with resident, faculty, and department leadership input was used to create guidelines for faculty and resident roles in common clinical scenarios.

\section{Results}

Institutional and regional mandates 
Large faculty gatherings and conferences were not permitted and were converted to online teleconferences according to university guidelines. In-person encounters between clinicians and patients were limited to the minimum necessary. Elective surgery and patient care activities were curtailed according to state mandate and medical center guidance, with a resultant significant decrease in clinical and surgical volume for trainees. Surgical teams were restructured.

\section{Team cohorting}

In order to limit patient exposure and team risk, we introduced a team cohort approach: a rotating smallteam approach designed for each clinical site in our residency program as described by other surgical subspecialties ${ }^{4}$. Each cohort team is prevented from physical interaction with the other teams and cover separate days in the hospital. Faculty-resident partnerships were assigned to limit interaction between Department members but provide comprehensive coverage at each clinical site. Residents were assigned to physician teams and they worked as a unit, being on call the same days and off the others. Attending teams were sequestered as well. This reduced the chance of spread of infection throughout the program should one resident or attending become infected.

\section{New paradigm for faculty and resident clinical care roles}

Continuing resident involvement in clinical activities is considered a critical aspect of ongoing resident education. This priority has been previously described by the head and neck surgery department in Lombardy, Italy ${ }^{5}$. The traditional hierarchy of junior residents performing primary assessment of patients guided by a senior resident is disrupted for two reasons. First the cohort system requires more manpower for daily assignments. Second, in order to reduce the number of at risk exposures, involvement of an experienced clinician is critical at the earliest phase of clinical interactions. In the new paradigm, detailed management of urgent otolaryngologic issues could be discussed as they arose through one-on-one discussion, in small groups, and in group video conferences, with special attention paid to the unique impact of the ongoing pandemic on clinical decision-making. Certain activities once considered routine for a head and neck surgeon, such as bedside endoscopy or tracheotomy change, now pose potential risk to the surgeon and trainee. Consideration is given to which patients require such procedures and who should be present. There were also opportunities to discuss evolving appropriate use and re-use guidelines as well as proper donning and doffing of personal protective equipment.

\section{Risk Stratification}

In order to define the working relationship for these small group partnerships, common otolaryngologic procedures were identified and risk-stratified based on existing evidence. Faculty and resident patient care roles were assigned based upon patient status, clinical care requirements, and mitigation of provider risk. Patients were categorized into three groups based on risk: COVID-positive patients, patients under investigation (PUI), and non-COVID/non-PUI. For this third category which may include asymptomatic carriers, a shared model of faculty/resident care was devised based upon provider experience and procedure risk.

Drawing from available evidence for the SARS-CoV-2 virus and related viral infections including SARS$\mathrm{CoV}$ and MERS-CoV,${ }^{6-10}$ clinical patient encounters and procedures were divided into low risk and high risk categories. Special consideration was given to procedures with high potential for viral transmission by aerosol generation: endoscopy (nasal and laryngoscopy) and tracheotomy (Table 1). Levels of faculty and resident involvement were defined as faculty only direct patient care, direct supervision, and consultation followed by indirect supervision (Figure 1).

\section{Discussion}

The field of Otolaryngology - Head and Neck Surgery has an intimate relationship with the primary anatomic regions associated with high viral load in the upper aerodigestive tract. With this realization comes a critical responsibility to ensure that we are not placing our trainees at risk in the name of education or as part of "business as usual." Early publications and social media interactions have highlighted the concerns for 
COVID-19 transmission in our field ${ }^{11-16}$. A full understanding of our roles as physicians, however, lays bare the fact that we cannot avoid risk.

Residents are routinely the front-line provider of care for inpatients and emergency department consultations. The new infectious risk associated with each clinical interaction threatens resident wellbeing - both physical and mental ${ }^{17,18}$. As a group, faculty are older and carry more comorbidities than residents, placing them at significantly higher risk if infected with the novel coronavirus. Faculty also carry the ultimate responsibility for both patient care and resident training and well-being; faculty are financially compensated for these responsibilities. Ultimately we are all physicians who have accepted the responsibility to care for our patients. We must also accept the responsibility to care for each other.

Treating patients during a pandemic setting has resulted in a significant reduction of existing resident learning opportunities such as in-person conferences, surgical volume and assessment of patients in clinic and on the floor. The cohorting system was implemented to diminish risk of spreading infection within our department, but also created an intimate team atmosphere with enhanced opportunity for individualized teaching. Each clinical encounter now results in a careful assessment of risk and determination of the appropriate extent of evaluation prior to performing an examination. During daily team discussions, a focus on the determinants of the optimal extent of evaluation for each patient provides an ideal opportunity for in-depth discussion of symptoms, pathology, diagnostic tests and treatment options.

While we are focused on trainee safety, we also recognize that restricting junior residents from traditional clinical activities may restrict their ascension to the next training level. The traditional approach to clinical training has the least experienced trainee performing the initial evaluation of a patient and reporting those findings to more senior team members who subsequently repeat the critical portions of the exam. Clearly, this repeated exposure is inappropriate for patients with known COVID disease ${ }^{19}$. Further, the significant rate of asymptomatic carriers of disease and limitations with availability and sensitivity of testing require changes to this protocol for all patient encounters. Development of guidelines which account for risks presented by both patients and clinical scenarios facilitates team care by identifying optimal care providers for each scenario.

The guidelines presented here are neither comprehensive nor universally applicable, but represent our experience at a major academic center at a time when viral testing and treatment are limited. There certainly will be specific patient scenarios in which clinical judgement demands deviation from guidelines. One example is a true airway emergency in which delay for the sake of guideline compliance could result in disastrous patient care consequences. The potential risk of evaluations as simple as oral cavity examination merits discussion with a faculty member prior to clinical interaction. In some scenarios, however, faculty approval may be implied or carried forward. With an inpatient following oral cavity resection and reconstruction, for example, daily examinations by the rounding team would be expected. The specifics of these guidelines are likely not appropriate for all programs. Variations in geography, practice settings, and most importantly endemic rates of COVID infection will dictate ideal guideline details for individual sites. Adapting and adopting guidelines for management of these common patient interactions, however, provides an opportunity to mitigate risk to the healthcare team without compromising the quality of patient care.

Our categorization of procedural risk was based on an estimation of the likelihood of aerosol generation and resultant short-distance airborne transmission of infectious viral particles during each procedure in question. While epidemiologic and experimental data is limited, studies from the 2002-2003 SARS outbreak support that there was a significant increased risk of transmission of infection to health care workers performing or involved in aerosol-generating procedures (AGPs) ${ }^{6,10}$. In particular, 2014 WHO guidelines note consistent evidence for increased risk with tracheal intubation, tracheotomy, non-invasive ventilation, and manual ventilation before intubation as AGPs based on work including a systematic review published in 2012 of ten low-quality studies from the SARS epidemic ${ }^{6,7}$. The data also support that there is a hierarchy of risk among aerosol-generating procedures included in these studies, which is reflected in our categorization ${ }^{6}$. Significantly, the viral load of SARS-CoV-2 is known to be very high in both the upper and lower respiratory tract in an infected patient, with somewhat higher viral loads in the nose than in the oropharynx ${ }^{20-22}$. We supplemented the conclusions of the above studies with additional cohort studies, case reports, expert 
opinion, and practical judgment in categorizing procedures for which either little to no data currently exists (e.g. nasal endoscopy, laryngoscopy, skull base surgery, control of epistaxis, and drainage of peritonsillar abscess) $)^{7,10,12,23}$.

Tracheotomy and endoscopic examinations are among those specifically called out as high risk procedures in our protocol. In the case of tracheotomy, there is consistent evidence for increased risk of viral transmission from the SARS epidemic ${ }^{7}$, presumably due to likelihood of aerosolization of a high concentration of virus for a prolonged period. Whenever possible, this procedure should be performed by the minimum number of experienced providers to complete the procedure expeditiously. Endoscopy, while frequently thought of as simply an extension of the Otolaryngologist's physical exam, carries significant risk for the clinician as well as staff and technicians who support the exam and process instrumentation. In our protocol, early faculty involvement is vital in evaluating the initial request or indication for the procedure, as they are in a position to efficiently and effectively decline this procedure in favor of alternative diagnostic evaluations whenever appropriate. In emergent or urgent scenarios where endoscopy is required, having faculty perform or directly supervise the exam ensures efficient and comprehensive evaluation in a single setting.

We have found that a small-team cohorting model with established guidelines delineating clinical roles and responsibilities can serve to strengthen the bond between faculty and residents. Strengthening this bond may be the most critical component of the COVID-19 response in residency training. Both faculty and residents are at risk; our early institutional data demonstrates a similar number of faculty and resident infections ${ }^{3}$. Recent resident surveys suggest that the amount of risk perceived by a resident correlates with the trust they have in their Department administration ${ }^{1}$. Faculty should be involved at the earliest stages of patient care including initial consultation. We are uniquely positioned to decline non-critical, aerosol-generating scope exams and identify streamlined evaluations to limit clinician exposure while maintaining patient care. When faculty join residents on the frontlines of patient care we model the physicianship central to managing a crisis, diminish risk to health care providers, and empower residents to remain engaged in meaningful patient care.

\section{Conclusions}

The COVID-19 pandemic has inexorably altered patient care for the Otolaryngologist - Head and Neck Surgeon. As we remain hopeful for a return to normalcy in residency training, adopting a small-team based approach and guidelines for patient exams and interactions has the potential to mitigate risk while preserving patient care and maximizing education.

\section{References}

1. Cai Y, Jiam N, Wai K, Shuman E, Roland L, Chang J. Otolaryngology Resident Practices and Perceptions in the Initial Phase of the U.S. COVID-19 Pandemic. 2020.

2. AAOHNS. Position Statement: Otolaryngologists and the COVID-19 Pandemic. https://www.entnet.org/content/otolaryn and-covid-19-pandemic. Published 2020. Accessed April 13, 2020.

3. UCSF. UCSF Health COVID-19 Clinical Update 4/10/2020. https://infectioncontrol.ucsfmedicalcenter.org/covid/ucsfhealth-covid-19-clinical-updates. Published 2020. Accessed April 13, 2020.

4. Schwartz AM, Wilson J, Boden SD, Moore TJ, Bradbury TL, Fletcher ND. Managing Resident Workforce and Education During the COVID-19 Pandemic Evolving Strategies and Lessons Learned. J Bone Jt Surg . 2020;0. doi:10.2106/JBJS.OA.20.00045

5. Saibene AM, Allevi F, Biglioli F, Felisati G. Role and Management of a Head and Neck Department during the COVID-19 Outbreak in Lombardy. Otolaryngol Head Neck Surg . 2020:194599820917914. doi:10.1177/0194599820917914

6. Tran K, Cimon K, Severn M, Pessoa-Silva CL, Conly J. Aerosol generating procedures and risk of transmission of acute respiratory infections to healthcare workers: A systematic review. PLoS One . 2012;7(4). doi:10.1371/journal.pone.0035797 
7. World Health Organization(WHO). Infection prevention and control of epidemic- and pandemic-prone acute respiratory infections in health care. WHO Guidel . 2014:1-156. http://apps.who.int/iris/bitstream/10665/112656/1/9 eng.pdf?ua $=1$.

8. World Health Organization(WHO). Modes of transmission of virus causing COVID-19 : implications for IPC precaution recommendations. https://www.who.int/news-room/commentaries/detail/modes-of-transmissionof-virus-causing-covid-19-implications-for-ipc-precaution-recommendations. Published 2020. Accessed April $12,2020$.

9. Siegel JD, Rhinehart E, Jackson M, Chiarello L and the HI, Committee CPA. Guideline for Isolation Precautions: Preventing Transmission of Infectious Agents in Healthcare Settings 2007 (CDC) .; 2007. https://www.cdc.gov/infectioncontrol/guidelines/isolation/index.html.

10. Raboud J, Shigayeva A, McGeer A, et al. Risk factors for SARS transmission from patients requiring intubation: A multicentre investigation in Toronto, Canada. PLoS One . 2010;5(5). doi:10.1371/journal.pone.0010717

11. Workman AD, Welling DB, Carter BS, et al. Endonasal instrumentation and aerosolization risk in the era of COVID-19: simulation, literature review, and proposed mitigation strategies. Int Forum Allergy Rhinol . 2020. doi:10.1002/alr.22577

12. Patel ZM, Fernandez-Miranda J, Hwang PH, et al. PRECAUTIONS FOR ENDOSCOPIC TRANSNASAL SKULL BASE SURGERY DURING THE COVID-19 PANDEMIC.Neurosurgery . 2020:19-22.

13. Vukkadala N, Qian ZJ, Holsinger FC, Patel ZM, Rosenthal E. COVID-19 and the otolaryngologist preliminary evidence-based review.Laryngoscope . 2020. doi:10.1002/lary.28672

14. Miravittles J. Current Otorhinolaryngology COVID-19 Literature Review. https://oto.med.upenn.edu/2020/03/31/curre otorhinolaryngology-covid-19-literature-review/. Published 2020. Accessed April 12, 2020.

15. Lu D, Wang H, Yu R, Yang H, Zhao Y. Integrated infection control strategy to minimize nosocomial infection of coronavirus disease 2019 among ENT healthcare workers. J Hosp Infect . 2020;(xxxx):2019-2020. doi:10.1016/j.jhin.2020.02.018

16. Cui C, Yao Q, Zhang D, et al. Approaching Otolaryngology Patients during the COVID-19 Pandemic.

17. Tsai C. Personal Risk and Societal Obligation Amidst COVID-19.JAMA J Am Med Assoc . 2020. doi:10.1001/jama.2020.5450

18. Bressman E. Should We Avoid Exposing Residents to Coronavirus? NEJM Journal Watch. https://blogs.jwatch.org/gene1 medicine/index.php/2017/11/thoughts-on-stigma/?loginprompt=social. Published 2020. Accessed April 12, 2020 .

19. Nassar AH, Zern NK, McIntyre LK, et al. Emergency Restructuring of a General Surgery Residency Program During the Coronavirus Disease 2019 Pandemic: The University of Washington Experience. JAMA Surg . 2020:4-7. doi:10.1001/jamasurg.2020.1219

20. Wölfel R, Corman VM, Guggemos W, et al. Virological assessment of hospitalized patients with COVID2019. Nature . 2020:1-10. doi:10.1038/s41586-020-2196-x

21. Yu F, Yan L, Wang N, et al. Quantitative Detection and Viral Load Analysis of SARS-CoV-2 in Infected Patients. Clin Infect Dis . 2020:1-19. doi:10.1093/cid/ciaa345

22. Tang Y-W, Schmitz JE, Persing DH, Stratton CW. The Laboratory Diagnosis of COVID-19 Infection: Current Issues and Challenges. J Clin Microbiol . 2020;(April):1-22. doi:10.1128/JCM.00512-20

23. Thompson KA, Pappachan J V., Bennett AM, et al. Influenza Aerosols in UK Hospitals during the H1N1 (2009) Pandemic - The Risk of Aerosol Generation during Medical Procedures. PLoS One . 2013;8(2). doi:10.1371/journal.pone.0056278 


\section{Hosted file}

Fig1 (1).docx available at https://authorea.com/users/312328/articles/444299-development-ofclinical-care-guidelines-for-faculty-and-residents-in-the-era-of-covid-19

\section{Hosted file}

Table1 (1) .docx available at https://authorea.com/users/312328/articles/444299-development-ofclinical-care-guidelines-for-faculty-and-residents-in-the-era-of-covid-19 